\title{
TITLE
}

\section{16th Iberian Conference on Information Systems and Technologies (CISTI)}

Proceedings of CISTI'2021 - 16th Iberian Conference on Information Systems and Technologies

23 to 26 of June 2021

Chaves, Portugal

\section{EDITORS}

Álvaro Rocha, Universidade de Lisboa, Portugal

Ramiro Gonçalves, Universidade de Trás-os-Montes e Alto Douro, Portugal Francisco Garcia Peñalvo, Universidad de Salamanca, Spain José Martins, AquaValor Research Center, Portugal

ISBN: 978-989-54659-1-0

WEB: http://www.cisti.eu/

CopyRight 2021: AISTI - Associação Ibérica de Sistemas e Tecnologias de Informação / Iberian Association for Information Systems and Technologies 


\section{MOOC sobre bullying para professores do $10^{\circ}$ ciclo do ensino básico}

\section{MOOC on bullying for primary school teachers}

\author{
Silvana Freitas Sousa \\ Escola Superior de Educação, Instituto Politécnico de \\ Bragança \\ Bragança, Portugal \\ silvanasousav@live.com.pt
}

\author{
Vitor Gonçalves \\ Centro de Investigação em Educação Básica, Instituto \\ Politécnico de Bragança \\ Bragança, Portugal \\ vg@ipb.pt
}

Resumo - O crescimento exponencial do bullying nas escolas contribuiu de forma significativa para que exista uma necessidade urgente de formação sobre esta temática, tanto para professores e pais, como também para alunos. Paralelamente, as mudanças tecnológicas a que assistimos na última década permitem-nos diversificar e adequar a formação às realidades atuais, incluindo os ambientes on-line onde o cyberbullying pode assumir contornos para os quais muitas crianças não estão preparadas. Assim, a criação de um MOOC parece a forma mais adequada e dinâmica para ensinar e prevenir o bullying em tempos de pandemia. Sustentado por um painel de experts, este estudo pretende responder de forma clara e objetiva às seguintes questões de investigação: i) Que tipos de MOOC existem no âmbito do bullying no Ensino Básico e respetivas plataformas para a sua distribuição? ii) Como é que este tipo de formação pode contribuir para a formação de professores? Que características podemos incluir no sentido de melhorar ou inovar a estrutura de um novo MOOC?

Palavras Chave - Bullying, ciberbullying, ensino básico, MOOC, formação de professores.

Abstract - The exponential growth of bullying in schools has contributed significantly to the urgent need for training on this topic, both for teachers and parents, as well as for students. At the same time, the technological changes that we have witnessed in the last decade allow us to diversify and adapt training to current realities, including online environments where cyberbullying can take on dimensions that many children are not prepared for. Thus, the creation of a MOOC seems the most appropriate and dynamic way to teach and prevent bullying in times of a pandemic. Supported by a panel of experts, this study aims to answer clearly and objectively the following research questions: i) What types of MOOC exist in the scope of bullying in Primary Education and the respective platforms for its distribution? How can this type of training contribute to teacher training? What characteristics can we include to improve or innovate the structure of a new MOOC?

Keywords - Bullying, cyberbullying, primary education, MOOC, teachers training.

\section{INTRODUÇÃO}

Cada vez mais o bullying é um importante tópico de estudo na vida das crianças e dos jovens, principalmente na última década. A importância advém do facto de que esta problemática tem vindo a assumir contornos de tal forma dramáticos para a vida dos alunos, pais e professores. $\mathrm{O}$ bullying é um ato que pode acontecer em qualquer lugar e a qualquer hora, e das mais variadas formas, desde violência física, verbal e psicológica bem como através do computador e das redes sociais, como é o caso do cyberbullying. Considera-se, pois, importante estudar o bullying no contexto dos professores do ensino básico, pois torna-se fundamental que a abordagem e prevenção da temática seja cada vez maior e em mais tenra idade. Por conseguinte, o estudo dos MOOC - Massive Open Online Courses - parece ser importante para perceber que tipo de formação existe para esta população e se ela está a ser lecionada de uma forma adequada e assertiva. Para tal, a revisão sistemática da literatura (RSL) foi a metodologia mais adequada para uma investigação qualitativa que pretende dar resposta às questões de investigação anteriormente referidas.

Esta RSL contribuiu para justificar a necessidade da conceção do MOOC StopIt! Este MOOC foi avaliado por um painel de 5 experts que, genericamente, considera que os conteúdos foram bem estruturados, que é possível aprender sobre o bullying através deste tipo de curso, mas que as apresentações beneficiariam se fossem mais dinâmicas e interativas.

\section{REVISÃo SISTEMÁTICA DA LITERATURA}

\section{A. Caracterização do Bullying}

Podemos definir bullying como uma agressão (física, verbal ou psicológica) praticada intencionalmente de forma a causar dor ou desconforto (físico ou psicológico) repetida ao longo do tempo e com desequilíbrio de poder entre os envolvidos (o agressor e a vítima). É importante sabermos distinguir os comportamentos de bullying de outros comportamentos igualmente agressivos, nomeadamente porque, em contexto escolar, ocorrem muitos outros comportamentos agressivos que não configuram uma situação de bullying.

Praticamente em toda a bibliografia da área, existem alguns critérios que nos ajudam nessa diferenciação e que importa aqui clarificar: a) Um comportamento de bullying, é sempre um comportamento agressivo intencional (não aconteceu por acaso nem sem querer, foi pensado/premeditado); b) É um comportamento sistemático, que assume um padrão de repetição ao longo do tempo (a frequência com que poderá ocorrer depende de caso para caso, mas nunca se trata de um incidente isolado, único); c) Implica uma desigualdade/abuso de poder 
entre os alunos envolvidos, o que implica que um deles se encontra numa posição de domínio/poder e o outro numa posição de impotência/submissão (pode ocorrer entre alunos mais velhos e mais novos, ou mais altos/fortes e mais baixos/fracos, etc.); d) Para que os dois anteriores critérios possam ocorrer (repetição e abuso de poder) é necessário que os envolvidos se encontrem com alguma regularidade. Por esse motivo, o bullying tende a ocorrer em grupos sociais que mantêm alguma familiaridade entre os seus elementos (tal como ocorre entre os alunos de uma turma que, ao final de um certo tempo de convivência, já conhecem as características e fragilidades uns dos outros).

Quanto às diferentes formas de bullying, também a bibliografia da área identifica os seguintes comportamentos: a) físicos, que implicam contacto físico, corporal, com o outro, como bater, empurrar, puxar os cabelos, dar pontapés, etc.; b) verbais, como chamar nomes ofensivos, insultar, rebaixar, fazer troça ou colocar alcunhas; c) relacionais, onde se manipulam as redes sociais e de amizade para, de forma indireta, atingirmos um terceiro elemento, como por exemplo fazer excluir um colega de um grupo ou de uma atividade, espalhar rumores utilizando os pares nessa disseminação, ou ignorar deliberadamente alguém; d) sexuais, que incluem todos os comportamentos agressivos de teor sexual, podendo os mesmos ser físicos ou verbais, como o exibicionismo, o assédio, os comentários ou insultos de natureza sexual, direcionados a partes do corpo ou relativos à orientação sexual; e) cyberbullying, quando os comportamentos de bullying utilizam como meio as tecnologias digitais, permitindo a rápida disseminação de conteúdos vários, através de todas as formas de comunicação que os vários dispositivos com acesso à Internet (telemóvel, tablet, computador) permitem.

Assim, podemos dizer que o bullying tem várias variantes e que de um modo geral pode trazer graves consequências se a escola, os professores e os pais não promoverem o diálogo e a formação, almejando maiores níveis de segurança para crianças e jovens.

\section{B. Caracterização dos $M O O C$}

Os Massive Open Online Courses (MOOC) são cursos online que normalmente estão disponíveis para qualquer pessoa. Os MOOC oferecem uma maneira acessível e flexível de aprender novos conteúdos, habilidades, progressão na carreira profissional e oferta de experiências educacionais de qualidade em grande escala (Ferri, D’Andrea, D’Ulizia, \& Grifoni, 2020).

Pelos registos de inscrições nas plataformas online de MOOC, podemos ver que há milhões de pessoas em todo o mundo que usam esta ferramenta para aprender por uma variedade de razões que incluem: o desenvolvimento ou mudança na área profissional, preparação para a universidade, aprendizagem suplementar, aprendizagem ao longo da vida, eLearning, formação corporativa entre outros (Ferri et al., 2020).

Os MOOCs mudaram, também, a maneira como o mundo aprende, pois através da internet e do computador é possível adquirir de forma rápida toda a formação que se precisa para os mais variados assuntos e tendo em conta que muita desta informação é fornecida pelas melhores e maiores instituições escolares do mundo. É possível ainda dar-se aulas ou formações completas para empresas e outros (Ferri et al., 2020). Durante esta pandemia por COVID-19 foi possível criar e disponibilizar MOOC para suportar ou complementar processos de aprendizagem diversos. Entre as abordagens de e-learning, o MOOC tem recebido muito interesse no que concerne à pesquisa educacional e tecnológica. São, por definição, cursos online gratuitos oferecidos a grandes massas através de ambientes de aprendizagem virtuais (Ferri et al., 2020). Portanto, atuam como agentes de mudança potencial no ensino de atividades de aprendizagem. A disseminação do MOOC precisa de atenção de todas as pessoas envolvidas em atividades de ensino e aprendizagem que tenham a oportunidade de melhorar as suas competências e habilidades.

Existem vários tipos de MOOC na literatura, contudo podemos agrupá-los em: a) cMOOC, ou MOOC conectivista, dá mais atenção à ligação entre os alunos do que a apresentação de conteúdo; b) xMOOC, este segue as estruturas de cursos tradicionais, faz uso de materiais e conteúdos pré-estabelecidos pelo autor ou formador e pode ser menos interativo; c) sMOOC, ou MOOC social incorpora um grande grau de interação social e participação diferente dos outros tipos de MOOC referidos.

Paralelamente ao modelo formal de ensino presencial, ou mesmo do ensino à distância mais tradicional surgem práticas de ensino online muito atrativas, com recurso, por exemplo, a vídeos e a objetos digitais interativos, observando-se o aumento da oferta formativa nessa modalidade (Costa, Santos, Guedes, \& Viana, 2015). A própria evolução crescente do uso das tecnologias digitais nas instituições de ensino superior e a produção de conteúdos e recursos educativos digitais distribuídos em plataformas de acesso livre facilitam e permitem a utilização simultânea de muitos utilizadores. Denota-se que estes conteúdos podem ser aplicados aos variados graus de ensino (básico e secundário) e que é preciso saber selecionar aqueles que são efetivamente de acesso livre. O contexto em que surge os Massive Open Online Course (MOOC), é mesmo o de uma oferta formativa vista como uma forte oportunidade de democratização no acesso ao conhecimento e que têm vindo a gerar um interesse considerável no ensino superior a nível mundial (Costa et al., 2015).

\section{Caracterização dos MOOC}

Os MOOC constituem, de facto, cursos que poderão envolver em simultâneo, dezenas de milhares de participantes considerando a facilidade de acesso online através da Internet e por serem disponibilizados (maioritariamente) de forma aberta e gratuita. Com esta vantagem facilmente incluímos os MOOC como formação para professores pois estes têm os mais variados conteúdos à disposição para a aplicabilidade nas aulas e adaptado ao estilo de cada um. Assim os MOOCs incluem um roteiro orientador das atividades a desenvolver, de forma a permitir que o aluno, que neste caso será o professor, conheça antecipadamente o percurso de aprendizagem, com indicação dos temas ou tópicos a tratar e respetivo cronograma. Com a disponibilização de um conjunto variado de recursos para acesso autónomo e independente aos conteúdos, onde recorrendo às potencialidades das tecnologias digitais e em rede, os MOOC integram ou disponibilizam conteúdos digitais abertos de diversas fontes, nomeadamente portais públicos, bancos internacionais de objetos de aprendizagem, e-books, entre outros (Costa et al., 2015). Pode contar, ainda, com a presença do 
professor instrutor na apresentação dos conteúdos, e na disponibilização de segmentos de vídeo como forma de substituição das aulas tradicionais e visando sobretudo manter o aluno interessado nos conteúdos a aprender (Costa et al., 2015). Para além disso, podem também ser desenvolvidas plataformas ou aplicações específicas, como a exploração de fóruns de discussão associados, administrados pelos professores instrutores em que os alunos colocam dúvidas ou discutem os conteúdos. Os mecanismos de voto permitem que as questões mais importantes sejam rapidamente visíveis pelos professores que podem assim esclarecer de uma só vez um grande número de alunos, para além de poder manter-se esse esclarecimento disponível para o futuro (Costa et al., 2015). Por último temos a inclusão de estratégias diversificadas de avaliação, que visam a confirmação se o aluno está ou não a compreender e a seguir o conteúdo exposto. Estas estratégias passam por questões que são colocadas no meio dos vídeos e às quais os alunos devem responder no momento. A avaliação do curso, dado o volume de alunos envolvidos, tanto recorre a processos automáticos, como aposta na exploração do potencial que resulta de os alunos avaliarem os seus próprios colegas, uma avaliação de pares que segue os critérios previamente estabelecidos (Costa et al., 2015). Assim concluímos que os MOOC são realmente uma potencialidade para formação de professores e para o ensino e que facilmente os docentes podem usar estes cursos para ministrar aulas e dar formação sobre conteúdos mais sensíveis como o que abordamos nesta investigação, o bullying. É de realçar a mudança de consciência que tem de existir por parte dos professores e das escolas para a adoção desta modalidade de ensino, que pelos resultados que mostra em nada vem prejudicar o trabalho habitual dos docentes.

\section{MOOC SOBRE BULLYING PARA O ENSINO BÁSICO}

Há uma necessidade enorme para que se forneçam cursos ou programas que criem um ambiente de aprendizagem saudável para os alunos, com um treino de conscientização que aborde o bullying e que inclua o aluno, a família e o professor de forma integrada, bem como a necessidade de estudos que avaliem os efeitos a longo prazo destes cursos ou programas. Estes programas permitirão que as escolas, as empresas e outras instituições trabalhem com direção a uma solução, aumentando assim a conscientização sobre o problema do bullying, bem como a diminuição das taxas do mesmo (Karatas \& Ozturk, 2020). Neste caso o objetivo é avaliar a existência de programas/cursos desenvolvidos para lidar com o bullying nas escolas primárias, por parte dos professores.

Pesquisas recentes mostraram que os antecedentes dos professores podem afetar suas perceções sobre o bullying. Por exemplo, professores com mais anos de experiência no ensino são menos propensos a simpatizar com as vítimas e podem até expressar atitudes negativas em relação a incidentes de bullying (Chen, Wang, \& Sung, 2018). No entanto, Burger, Strohmeier, Sprober, Bauman e Rigby (2015) descobriram que professores com mais de 25 anos de experiência no ensino relataram uma maior probabilidade de lidar com os incidentes de bullying, ajudando as vítimas através do psico-trauma lógico, e no melhoramento do comportamento de um bully em comparação com os outros professores inexperientes. Goryl, Neilsen-Hewett e Sweller (2013) exploraram a relação entre a experiência de ensino dos professores e sua confiança na capacidade de identificar incidentes de bullying e não encontraram correlação entre essas variáveis (Chen, Wang, \& Sung, 2018).

Assim, denotam-se três aspetos importantes. O primeiro é se os professores com experiências de ensino diferente podem ter dificuldades na identificação dos diferentes tipos de bullying. A segunda é se o género pode ser dificultador na identificação de casos de bullying. E em terceiro, se as diferenças académicas (habilitações) entre os professores pode ser motivo de dificuldade na identificação dos tipos de bullying (Chen, Wang, \& Sung, 2018).

Deste modo, considera-se a formação de professores com MOOC fundamental pois pode dar cobro aos aspetos acima referenciados. A formação ministrada de igual forma a todos os docentes faz com que os mesmos fiquem em conformidade relativamente à preparação e aos conhecimentos adquiridos para trabalhar com os casos de bullying. Além disso, os MOOC sobre esta temática podem auxiliar os gestores educacionais (escola) a julgar quais os cursos de formação que devem ser fornecidos para que grupos de professores com formação específica variável possam melhorar o seu desenvolvimento profissional de identificação e intervenção no bullying (Chen, Wang, \& Sung, 2018).

A maior parte dos trabalhos, em MOOC, produzidos para o bullying resultam das interações, reflexões e partilhas sobre um conjunto de conteúdos disponibilizados pelo orientador e participantes, sendo que este primeiro atua sobretudo como agente facilitador das aprendizagens, fornecendo apoio e promovendo a partilha e a discussão entre os participantes (Pedro \& Baeta, 2016).

Em particular, denota-se que, apesar de caber aos orientadores a função de fornecer os recursos de aprendizagem e indicar todas as tarefas a realizar e procedimentos a seguir, deve haver uma linha orientadora a seguir que já foi referida no tópico, Massive Open Online Courses como formação para professores em educação de crianças e jovens.

Assim e apesar do modelo pedagógico dos MOOC geralmente se destacarem por não conter somente conteúdos fixos para serem apreendidos, neste caso específico deve haver uma base fixa de conteúdos que é transversal à aprendizagem do bullying e que facilitará o reconhecimento e a identificação deste tipo de situações por parte dos professores. Este processo permitirá ao docente a partilha de conhecimento e prevenção para com toda a comunidade escolar e respetivos pais.

\section{Metodologia}

Esta pesquisa foi desenvolvida através de uma metodologia baseada numa revisão sistemática da literatura e num estudo caso que tem por base a conceção de um MOOC intitulado StopIT - Bullying para Professores do Ensino Básico. A RSL permitiu uma investigação sobre o que é o bullying e as suas variantes de uma forma geral bem como a investigação da formação em MOOC que existe sobre esta temática para docentes. Com recurso a algumas bases de dados, como o Web of Science, Scopus e Google Scholar, pesquisaram-se artigos científicos relacionados com os problemas desta investigação. Após a criação do curso MOOC e de este ter sido publicado procedeu-se à avaliação do mesmo por um painel de experts. Esta avaliação consistiu num questionário que se divide pelos 
vários tipos de estrutura do MOOC no caso, os conteúdos do MOOC, os Parâmetros Estruturais, Funcionais e das Percepções. A escala de avaliação do questionário é de Likert correspondente a 0,5 e 10 , como 0 - não possuí; 5 - possuí parcialmente e 10 possuí totalmente. Este tipo de classificação permitirá identificar a capacidade avaliativa e atenção dos experts a toda a composição do curso MOOC.

\section{Resultados: Estudo CASO - MOOC StopIT}

Os resultados que aqui se apresentam pertencem à avaliação correspondente ao curso MOOC preenchida por um painel de experts, através de um questionário do Google Forms dividido em secções para a avaliação dos seguintes parâmetros, conteúdos do MOOC e parâmetros estruturais, funcionais e das percepções. Entre cada secção havia perguntas específicas sobre alguns conteúdos do curso, a fim de percebermos se o respetivo painel tinha de certa forma percorrido todas as componentes do curso.

Como já referido, o questionário de avaliação foi aplicado a um painel de cinco experts, este era constituído por Professores, Psicólogos e Estudantes ao nível do Mestrado e Doutoramento nas áreas das TIC, Educação, Formação de Professores, Educação Social e Comportamentos desviantes.

A este questionário a escala de avaliação correspondente era a de lickert, com os valores de 0,5 e 10 , como anteriormente referido, 0 é igual a não possui; 5 a possui parcialmente e 10 possuí totalmente. A média dos resultados é de 8 o que é bastante positivo para o efeito e para o tempo disponível para a concepção do curso e respetiva avaliação. Como pontos a melhorar no curso fica a diversificação das tarefas e exercícios, de modo a tornálos mais objetivos e variados, bem como a necessidade de colocar as VideoAula mais apelativas, a fim da sua visualização não ser considerada cansativa.

\section{A. Interpretação dos dados: Conteúdo do MOOC}

Esta $1^{a}$ secção do questionário consistia na verificação, por parte dos experts, da existência clarificada no curso dos objetivos e estratégias de aprendizagem; da aplicabilidade correta das temáticas; da existência de elementos multimédia; de atividades e exercícios; da articulação com outras plataformas; da informação referenciada e correta e da qualidade das Vídeo Aulas. Assim de acordo com o gráfico nota-se os seguintes resultados.

Figure 1. Gráfico dos resultados do conteúdo do MOOC

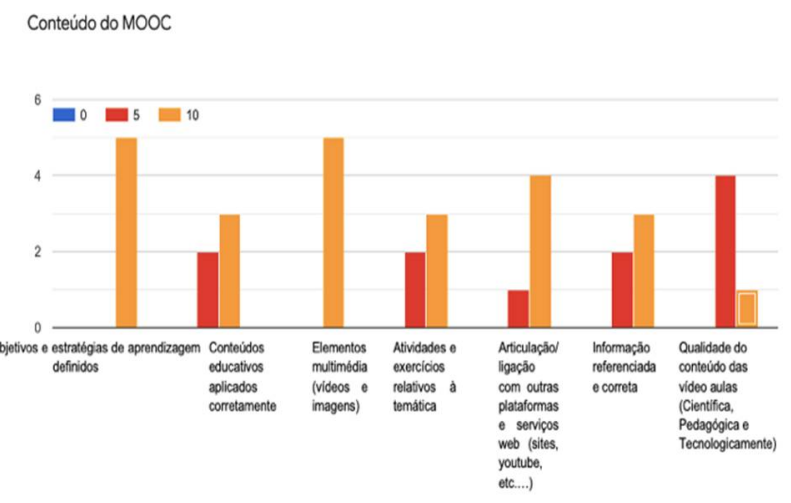

\section{Parâmetros Estruturais}

Esta $2^{\mathrm{a}}$ secção corresponde aos parâmetros que abordam a interação entre participantes; este aspecto avalia o nível de interação entre os alunos do MOOC, levando em consideração as ferramentas disponíveis para tal e o estímulo dado pelos professores a esta interação; Realização assíncrona (flexibilidade): relacionada com atendimento das necessidades de aprendizagem em momentos e ritmos diferentes. Cada aluno tem o seu "tempo de aprendizagem" e a plataforma deve respeitar isso e permitir que todos tenham condições para a conclusão do curso; Aprendizagem autónoma e em rede (conectividade): outra característica fundamental do conectivismo. A aprendizagem autónoma deve ser compartilhada na rede, discutida, avaliada e transformada, criando assim uma aprendizagem coletiva;

Assim, para o campo da interação o grupo de experts ficou divido sendo que 1 considerou que não havia interação entre os participantes; 2 consideraram que havia de forma parcial e os últimos 2 de forma total. Entende-se que a divergência de opiniões seja pela falta de percepção de como é realizada a interação entre grupos neste tipo de cursos MOOC.

Relativamente à aprendizagem autónoma, esta foi unânime pelo painel, pois sabemos de antemão que este tipo de formação é baseado em conteúdos devidamente organizados e expostos que seguem uma determinada estrutura e que é possível o acompanhamento dos formandos, mas que a maioria da aprendizagem é feita de forma autónoma e independente.

\section{B. Interpretação dos dados: Parâmetros Funcionais}

À secção 3 dos parâmetros funcionais correspondem as aulas em formato de vídeo: trata-se do aspeto básico que caracteriza os MOOC; download de vídeo: característica relevante, uma vez que o aluno pode fazer o download do conteúdo e estudar em locais onde, eventualmente, não haja acesso à internet ou haja restrição de acesso. Sugestão de materiais de apoio: os materiais de apoio servem para o aprofundamento dos aspectos de interesse do aluno, além de servirem como instrumento de consulta para as eventuais atividades. Quadro de performance do aluno: quadro geral onde é apresentada a performance discente, em relação à visualização de conteúdos, realização de atividades, participação nos fóruns e outros aspectos que consideram ser relevantes; Emissão de certificado: certificados, normalmente, são conferidos por meio de pagamento de uma taxa, definida pela plataforma, e, geralmente, estão condicionados à realização de uma avaliação final e autoavaliação ou avaliação de aprendizagem.

Respetivamente à avaliação dos experts para estes parâmetros conclui-se que, para a maioria estes aspetos foram cumpridos na totalidade, ou seja 4 em 5 atribuem a avaliação de 10, à exceção da certificação, que contou com uma classificação de 2-10, 2-5 e 1-0. A certificação do curso só é possível de se obter se todas as etapas do curso forem concluídas, o que se depreende que esta divisão estará relacionada com o progresso de cada expert no curso. Ou seja, se não o realizaram na totalidade, não obtiveram a certificação final. Conclui-se também a avaliação desta secção foi a mais unânime. 


\section{Interpretação dos dados: Parâmetros das Percepções}

À $4^{\mathrm{a}}$ e última secção correspondem a facilidade de navegação: está relacionada com o quão intuitiva é a navegação na plataforma, ou seja, para um utilizador leigo, qual seria o nível de dificuldade em aceder às funcionalidades. A qualidade dos vídeos: refere-se ao nível de apresentação dos mesmos, ou seja, se são apenas reprodução de slides, se há participação efetiva dos professores e se possuem ambientes diversificados e efeitos que podem auxiliar o aluno na compreensão dos conteúdos. Clareza e objetividade na linguagem empregada: pelo facto dos MOOC serem destinados ao público em geral, a linguagem deve ser empregue da forma mais simples e objetiva, sem perder o rigor científico que o tema requer, mas sem excessos que impeçam a compreensão por parte da maioria das pessoas.

Quanto à avaliação, o quadro seguinte ilustra a opinião dos experts.

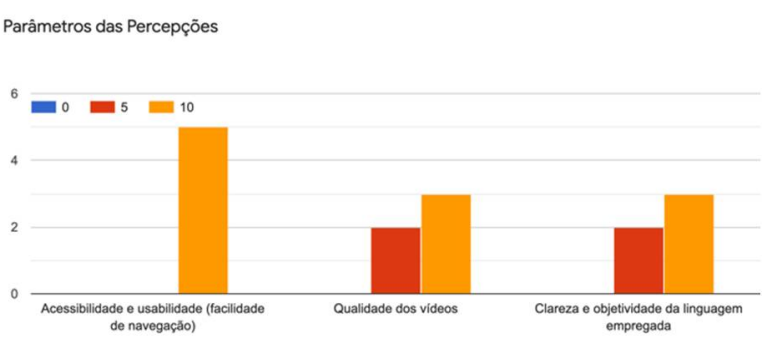

Figure 2. Gráfico dos resultados dos Parâmetros das Percepções.

Conclui-se assim que avaliação no seu âmbito geral considera que os conteúdos foram bem estruturados, que é possível aprender sobre o bullying através destas aulas, mas que as apresentações beneficiariam se fossem mais dinâmicas. Considera-se ainda, que para um primeiro curso de Bullying junto da comunidade escolar este conteúdo é oportuno, especialmente para suscitar curiosidade e interesse. Deste modo, os professores podem mais facilmente identificar situações específicas e partilhar experiências ente si, ou até mesmo, caso necessitem, solicitar apoio.

\section{CONCLUSÕES}

Ao longo do trabalho aqui desenvolvido fui possível descobrir prováveis causas para o acontecimento do Bullying nas escolas, e alguns referem a falta de perceção e descoberta de professores e pais sobre o que acontece com os seus alunos e filhos, respetivamente. $\mathrm{O}$ que pode corresponder à falta de conhecimento sobre a temática e a forma como ela se perpétua na vida dos alunos, seja as vítimas, sejam os agressores. Assim, urge a necessidade de cada vez mais termos professores preparados para lidar com estas situações, bem como, meios disponíveis para absorção desse conhecimento como é o exemplo do curso MOOC aqui criado. O projeto, em si, foi desenvolvido dentro das datas estabelecidas e cumprindo as tarefas que cada fase compelia. A criação dos conteúdos em vídeo para o curso foi notoriamente o que deu mais trabalho, visto que tinha de ser elaborado com o máximo rigor para garantir a qualidade do material que ia ser publicado. Quanto à avaliação do MOOC pelo painel de experts podemos concluir que a iniciativa de criação do MOOC está muito boa e que para uma primeira abordagem ao tema, os conteúdos fornecidos são os que se pretendem, devendo apenas diversificar um pouco mais as tarefas e questionários aplicados, bem como alguns conteúdos em vídeo. Os dados provenientes da avaliação são no âmbito geral de fácil percepção, contudo a escala escolhida poderá não ter sido a mais adequada pois uma de 1 a 5 tinha-nos permitido a obtenção de maior amplitude e clareza de opiniões.

Em suma, a criação deste curso MOOC serviu para percebermos que é possível utilizar este tipo de modelo tecnológico para formação, pois assim esta torna-se mais apelativa e significativa; pode ser feita a qualquer altura e em casa, o que é ideal nestes tempos de pandemia e que através da plataforma escolhida é possível criar este tipo de cursos com as mais variadas estruturas e conteúdos.

\section{REFERÊNCIAS BIBLIOGRÁFICAS}

[1]. Almeida, K. L., Cavalcante, A., \& Silva, J. S. C. (2008). Importância da identificação precoce da ocorrência do bullying: uma revisão de literatura The importance of early identification of bullying: a review of the literature. Revista de Pediatria, 9(1), 8-16.

[2]. Baeta, P. I. P. (2016). Moocs desenvolvidos no ensino superior Português: análise de modelos pedagógicos e estratégias de funcionamento utilizados (Doctoral dissertation).

[3]. Costa, F. A., Santos, A. M., Guedes, A., \& Viana, J. (2015). Guiões para desenho de cursos mooc, 327-342. Retrieved from http://groups.ist.utl.pt/ mooclab.daemon/wpcontent/uploads/2015/09/2015COSTA-et-al.-Guiõespara-desenho-de-Mooc-Livro.pdf

[4]. Chen, L. M., Wang, L. C., \& Sung, Y. H. (2018). Teachers' recognition of school bullying according to background variables and type of bullying. Journal of Educational, Cultural and Psychological Studies, (18), 147-163. https://doi.org/10.7358/ecps-2018018-chen

[5]. Ferri, F., D’Andrea, A., D’Ulizia, A., \& Grifoni, P. (2020). Co-Creation of e-learning Content: The Case Study of a MOOC on Health and Cyber-Bullying. Journal of Universal Computer Science, 26(2), 20. https://doi.org/10.1111/cjag.12228

[6]. Karatas, H., \& Ozturk, C. (2020). Examining the Effect of a Program Developed to Address Bullying in Primary Schools. The Journal of Pediatric Research, $7(3)$,

243-249. https://doi.org/10.4274/jpr.galenos.2019.37929

[7]. Pedro, N., \& Baeta, P. (2016). Balanços de vidas, medo da morte e esperança na salvação.pdf. Indagatio Didactica, 8, 128-152. 